\title{
Emotional, Physical, and Sexual Child Abuse in China: Prevalence and Psychological Consequence
}

\author{
Michelle Jin, Qian Wang, Xiaojing Xu, Jie Zhong \\ School of Psychological and Cognitive Science, Beijing Key Laboratory of Behavior and Mental Health, Peking University, Beijing, \\ China \\ Email: jzhong@pku.edu.cn
}

How to cite this paper: Jin, M., Wang, Q., $\mathrm{Xu}, \mathrm{X}$. J., \& Zhong, J. (2021). Emotional, Physical, and Sexual Child Abuse in China: Prevalence and Psychological Consequence. Psychology, 12, 1325-1340. https://doi.org/10.4236/psych.2021.128083

Received: July 21, 2021

Accepted: August 22, 2021

Published: August 25, 2021

Copyright ( 2021 by author(s) and Scientific Research Publishing Inc. This work is licensed under the Creative Commons Attribution International License (CC BY 4.0).

http://creativecommons.org/licenses/by/4.0/ (c) (i) Open Access

\begin{abstract}
The present study aimed to examine different degrees of child abuse and related psychological consequences in the mainland of China. A Chinese version of the Norvold Abuse Questionnaire was administered to 981 Chinese college students. The prevalence rates of child emotional abuse were $16.4 \%$, $8.8 \%$, and $6.9 \%$ for the mild, moderate, and severe levels of abuse, respectively, and those of physical abuse were $34.3 \%, 19.3 \%$, and $3.1 \%$, respectively. Furthermore, $4.4 \%$ of the participants in the mild sexual abuse group reported having had no genital contact and $1.0 \%$ of them reported having experienced emotional humiliation. The prevalence rates of moderate and severe sexual abuse were $2.4 \%$ and $.8 \%$. More males were found to suffer from moderate or severe levels of emotional abuse than females. Similarly, more males reported physical abuse than females across all degrees of severity. Mild and severe physical abuse had a larger current effect on males than on females, whereas the impact of moderate physical abuse was greater on females. Overall, Chinese college students reported significantly fewer sexual abuses than similar samples in Western countries. Additionally, more females suffered from mild sexual abuse than males. These findings suggest a potential influence of gender and culture.
\end{abstract}

\section{Keywords}

Child Abuse, Prevalence Rates, Current Effects, Gender, Culture

\section{Introduction}

Child abuse is psychological, sexual, and physical maltreatment or neglect of a child or children, especially by a parent or a caregiver (Runyan et al., 2002). The 
World Health Organization defines child abuse and child maltreatment as all forms of physical and/or emotional ill-treatment, sexual abuse, neglect, or negligent treatment, or commercial or other exploitation, resulting in actual or potential harm to the child's health, survival, development, or dignity in the context of a relationship of responsibility, trust, or power (Runyan et al., 2002). Maltreated children may have severe life-long behavioral, cognitive, neurological, and psychological or psychiatric problems that persist through adulthood (Corsello et al., 2016; Currie \& Widom, 2010; Hughes et al., 2009; Jaffee \& Maikovich-Fong, 2011; Norman et al., 2012).

Emotional, physical, and sexual abuse are three distinguished subtypes of child abuse that may lead to enduring negative effects on children's neural, physical, and psychosocial development (Krug et al., 2002; Luke \& Banerjee, 2013). Child emotional abuse, also known as psychological abuse, includes six major categories of parental behavior: spurning, terrorizing, isolating, exploiting, denying responsiveness, and mental health, medical care, or legal neglect (Brassard \& Donovan, 2006). It is regarded as an insidious and psychologically damaging form of child maltreatment that may result in psychological problems like low self-esteem, anxiety, depression, unhealthy dependency, and self-destructive behaviors (Calvete, 2014; Kane \& Bornstein, 2018; U.S. Department of Health Human Services, 2005; Wright et al., 2009). Child physical abuse is a form of frequent intentional infliction of physical harm or injury on a child, including hitting, kicking, beating, shaking, biting, strangling, scalding, suffocating, poisoning, and burning (Shaffer et al., 2008). Individuals who were subjected to physical abuse in child may suffer a higher risk of mental health problems such as depressive disorders, eating disorders, and child behavioral disorders (Norman et al., 2012; Shaffer et al., 2008; Springer et al., 2007), suicidality (Pompili et al., 2009), and psychotherapy attrition (Chasson et al., 2013). Child sexual abuse refers to involving children in sexual activities they do not understand or are not developmentally prepared for, and for which they are unable to consciously decide whether to participate (Shaffer et al., 2008). Child sexual abuse victims may be at a greater risk of having thought and language disruptions, adverse sexual health outcomes, suicidal or self-injurious behaviors, and mental disorders, such as major depressive disorder, phobic disorders, and generalized anxiety disorder (Amado et al., 2015; Conway et al., 2013; Lacelle et al., 2012; Maniglio, 2011; Oz \& Ogiers, 2006).

Previous studies have shown a high prevalence rate of child abuse across the world (Hillis et al., 2016; Madu, 2001; Stoltenborgh, Bakermans-Kranenburg, \& Van, 2013; United Nations Children's Fund, 2012). The global prevalence rate of child abuse was estimated to be $26.7 \%$ (95\% CI: $14.4 \%$ - 44.2\%) emotional, $22.6 \%$ (95\% CI: $19.6 \%$ - 26.1\%) physical, and 11.8\% (95\% CI: $10.0 \%-13.8 \%)$ sexual (Stoltenborgh, Bakermans-Kranenburg, \& Van, 2013). In Asia, 41.6\% (95\% CI: $23.3 \%-62.5 \%$ ) of Asian children have suffered from emotional abuse, $16.7 \%$ (95\% CI: 10.6\% - 25.3\%) physical abuse, and 11.3\% (95\% CI: 7.5\% - 16.6\%) sexual abuse (Stoltenborgh et al., 2012; Stoltenborgh, Bakermans-Kranenburg, Van, 
\& Alink, 2013; Stoltenborgh et al., 2011). Based on statistics from 68 studies in China, about $19.6 \%$ of Chinese children under the age of 18 have suffered emotional abuse, $26.6 \%$ physical maltreatment, and $8.7 \%$ sexual abuse (Fang et al., 2015).

Gender is considered an important factor influencing prevalence rates and psychological consequence of child abuse. Prevalence rates for child abuse under broad definitions (contact abuse) are higher for girls than for boys, with $20.4 \%$ versus $16.2 \%$ in Ireland, $15.8 \%$ versus $6.7 \%$ in Denmark, and $7.8 \%$ versus $3.2 \%$ in Greenland (McElvaney \& Lalor, 2014). Gender differences are distinct in child sexual abuse with a higher prevalence among girls than among boys (Lalor \& McElvaney, 2010; Pereda et al., 2009a, 2009b). An international review of studies conducted in 22 countries worldwide shows that $19.7 \%$ of women (19.2\% without outliers) and $7.9 \%$ of men (7.4\% without outliers) had suffered some form of child sexual abuse (Pereda et al., 2009b). In a meta-analysis on child sexual abuse including 331 studies worldwide, significant gender differences were found in Asia, Australia, Europe, and the United States/Canada, with girls showing a higher prevalence of child sexual abuse than boys (Stoltenborgh et al., 2011). Regarding the effects, females showed significantly higher rates of developing depressive disorder ( $42 \%$ over baseline) and/or anxiety disorder ( $24 \%$ over baseline) (Amado et al., 2015).

Besides gender, the geographical-cultural factor also has impacts on child abuse prevalence rates. Significant differences were found between continents of origin of the sample for both girls and boys. The estimated child sexual abuse prevalence rates for boys/girls in different regions were $4.1 \% / 11.3 \%$ in Asia, $13.8 \% / 13.4 \%$ in South America, 5.6\%/13.5\% in Europe, 19.3\%/20.1\% in Africa, $8.0 \% / 20.2 \%$ in the USA/Canada, and $7.5 \% / 21.5 \%$ in Australia (Stoltenborgh et al., 2011). It is noteworthy that child sexual abuse is asymmetrically distributed globally, with the lowest prevalence in Asia for both genders. Moreover, the prevalence rates in different countries within one continent are different. In the $\mathrm{UK}$, serious maltreatment was experienced by $6 \%$ of respondents for emotional abuse, $7 \%$ for physical abuse, and $11 \%$ for sexual abuse involving contact (May-Chahal \& Cawson, 2005). In a Norwegian mother and child cohort study, the prevalence rates of emotional, physical, and sexual abuse in child were $14 \%$, $6 \%$, and 7\% respectively (Sorbo et al., 2013). Furthermore, different prevalence rates are found among different ethnicities within one country. According to a study of 5,689,900 American children, 12.5\% (95\% CI, 12.5\% - 12.6\%) of children have experienced child maltreatment. The highest prevalence was found among Black (20.9\% [95\% CI, 20.8\% - 21.1\%]) children, and the lowest prevalence was found among Asian/Pacific Islander (3.8\% [3.7\% - 3.8\%]) children (Wildeman et al., 2014).

In China, child abuse is a pervasive and serious social problem without enough attention in either academia or society (Fang et al., 2015; Dunne et al., 2008). Partially due to the lack of validated research instruments, only a limited number of studies on child abuse have been conducted among the Chinese pop- 
ulation, especially in mainland China (Kim et al., 2000). The paucity of comprehensive statistics on the prevalence of major forms of child abuse and on the long-term ill influence of such maltreatments would hinder the development of child protection policies in China. Therefore, the present study selected the Norvold Abuse Questionnaire (NorAQ) to assess the prevalence and current effects of child abuse. The NorAQ has been adapted to clearly define and quantify abuse, and used as a validated measuring instrument in epidemiological surveys among several samples (Wijma et al., 2003).

To sum up, the current study aimed to investigate in detail the prevalence rates of child abuse and current effects in mainland China through the adapted Chinese version of Norvold Abuse Questionnaire (NorAQ). Specifically, three types of child abuse (emotional, physical, and sexual abuse) for three degrees of severity (mild, moderate, and severe) were examined. Furthermore, we explored the roles of gender and Chinese culture. Our research would reveal the status quo of child abuse in mainland China and offer quantitative evidence for the establishment and implementation of future abuse prevention and child protection programs in the country.

\section{Method}

\subsection{Participant}

A cross-sectional survey was conducted among 981 Chinese college students. Sixteen copies of questionnaires were incomplete and another three respondents did not report their gender. Therefore, 19 cases were excluded. The number of valid cases was 962; 555 (57.7\%) were from female respondents, and 407 (42.3\%) were from male respondents. The age of participants ranged from 17 to 23 years old ( $M=19.69, S D=1.12 ; 5$ participants did not report their age).

\subsection{Instrument}

The questionnaire administered in the present study was translated from the Norvold Abuse Questionnaire (NorAQ). The original NorAQ has eight parts consisting of 80 questions, and has been applied to patients visiting gynecology clinics in some North European countries (Wijma et al., 2003). Only the 4 - 6 parts on topics of emotional, physical, sexual abuse represented by 10 questions were selected as items of the Chinese version. The selected parts in the original NorAQ have good test-retest reliability ranging from $84 \%-95 \%$ and validity (Wijma et al., 2003). The NorAQ's specificity was $98 \%$ for emotional and sexual abuse except physical (85\%), and its sensitivity ranged from $75 \%$ for emotional abuse to $96 \%$ for physical (Swahnberg \& Wijma, 2003). All the selected items were translated, back-translated, evaluated, adapted, and finalized by two Chinese clinical psychologists ensuring that the questions were easy to understand for Chinese participants.

The Chinese version of NorAQ includes four parts. Part one has demographic questions about gender, age, place of birth, ethnic group, main occupation, and 
family income; the students were also asked to provide information regarding their year of study and major. The following parts are three structured sections on emotional, physical, and sexual abuse. The presence or absence of emotional and physical abuse is probed by three questions. Each question corresponds to the severity of the abusive experience (mild, moderate, and severe). Four questions concern sexual abuse experiences with two mild abuse questions (no genital contact, emotional sexual abuse), one moderate sexual abuse question (genital contact), and one severe sexual abuse question (penetration).

Answer categories for all questions are: $1=n o ; 2=$ yes, as a child $(<18$ years); $3=y e s$, as an adult ( $\geq 18$ years); $4=y e s$, as a child and as an adult. Emotional, physical, and sexual abuse are defined as choosing yes to one or several of the questions on each type of abuse. Participants who report more than one degree of a specific type of abuse are counted only once when estimating the total prevalence rates. If a participant scored 2 to 4 for any question, he/she would be asked to answer more detailed questions and to estimate to what extent he/she was currently affected by the abusive experience on a scale of 0 to $10(0=n o$ effect, 10 = serious effect $)$ (Wijma et al., 2003).

\subsection{Procedure}

The participants were asked to fill out the four-part Chinese version of NorAQ in class. The questionnaire packet contained a cover for the purpose of protecting the participants' privacy with a declaration of confidentiality and a statement that the questionnaire was for research purposes only. No identifying information, such as name, telephone number, or address was solicited. A short introduction was given on the cover offering guidance for completing the questionnaire and soliciting honest answers. If the questions aroused any difficult emotions, the participant was encouraged to contact the researchers directly by email or phone for professional help.

\subsection{Data Analyses}

Descriptive analyses were carried out with SPSS 24 . The child abuse odds ratio (OR) was represented by the percentage of people who reported child abuse experiences divided by the total number of males or females included in the current study. Pearson's Chi-square test (two sided) was performed to assess differences between the male and female participants, and among the three types of child abuse experiences.

\section{Results}

A total of 981 questionnaires were sent out and 962 copies were completed; the valid return rate was $98.1 \%$. Among the 962 participants, 158 reported mild emotional abuse experiences in their childhood (a prevalence rate of 16.4\%); 85 (8.8\%) reported moderate childhood emotional abuse experiences; and 66 (6.9\%) reported severe childhood emotional abuse. After eliminating repeating counting data, alto- 
gether $213(22.1 \%)$ reported to be emotionally abused before 18-year-old. More males experienced moderate $(12.3 \%$ vs. $6.3 \% ; p<.01)$ and severe $(9.3 \%$ vs. $5.1 \% ; p$ $<.01)$ emotional abuse than females. However, no gender difference was found in mild emotional abuse experiences. The results are summarized in Table 1.

Three hundred and thirty (34.3\%) respondents reported mild physical abuse experiences; 186 (19.3\%) participants reported moderate physical abuse, and 32 (3.1\%) reported having suffered severe physical abuse; these experiences also happened predominantly before the age of 18 . In total, 371 (38.6\%) reported to be physically maltreated when they were children. Men were more exposed to all categories of physical abuse than women (mild: $43.7 \%$ vs. $27.4 \%$; moderate: $28.0 \%$ vs. $13.0 \%$; severe: $6.1 \%$ vs. $.9 \%$; See Table 2 ). The above three gender differences were statistically significant $(p<.01)$.

While $42(4.4 \%)$ respondents reported mild child sexual abuse without genital contact, $10(1.0 \%)$ participants reported mild child sexual abuse consisting of emotional or sexual humiliation. Moderate sexual abuse with genital contact was reported by $23(2.4 \%)$ respondents and $8(.8 \%)$ respondents have experienced severe sexual abuse such as penetration. No gender difference was identified in any of the sexual abuse categories (See Table 3).

Table 1. Prevalence rate of child emotional abuse.

\begin{tabular}{cccc}
\hline \multirow{2}{*}{ Gender } & \multicolumn{3}{c}{ Severity of abuse } \\
\cline { 2 - 4 } & Mild & Moderate & Severe \\
\hline Male & $82(20.2 \%)$ & $50(12.3 \%)^{* *}$ & $38(9.3 \%)^{* *}$ \\
Female & $76(13.7 \%)$ & $35(6.3 \%)$ & $28(5.1 \%)$ \\
Sum & $158(16.4 \%)$ & $85(8.8 \%)$ & $66(6.9 \%)$ \\
\hline
\end{tabular}

Note. ${ }^{*} p<.05 ;{ }^{* *} p<.01$.

Table 2. Prevalence rate of child physical abuse.

\begin{tabular}{cccc}
\hline \multirow{2}{*}{ Gender } & \multicolumn{3}{c}{ Severity of abuse } \\
\cline { 2 - 4 } & Mild & Moderate & Severe \\
\hline Male & $178(43.7 \%)^{* *}$ & $114(28.0 \%)^{* *}$ & $27(6.1 \%)^{* *}$ \\
Female & $152(27.4 \%)$ & $72(13.0 \%)$ & $5(.9 \%)$ \\
Sum & $330(34.3 \%)$ & $186(19.3 \%)$ & $32(3.1 \%)$ \\
\hline
\end{tabular}

Note. ${ }^{*} p<.05 ;{ }^{* *} p<.01$.

Table 3. Prevalence rate of child sexual abuse.

\begin{tabular}{ccccc}
\hline \multirow{4}{*}{ Gender } & \multicolumn{4}{c}{ Severity of abuse } \\
\cline { 2 - 5 } & $\begin{array}{c}\text { Mild } \\
\text { (no genital contact) }\end{array}$ & $\begin{array}{c}\text { Mild } \\
\text { (emotional/sexual humiliation) }\end{array}$ & $\begin{array}{c}\text { Moderate } \\
\text { (genital contact) }\end{array}$ & $\begin{array}{c}\text { Severe } \\
\text { (penetration) }\end{array}$ \\
\hline Male & $18(4.4 \%)$ & $6(1.5 \%)$ & $12(3.0 \%)$ & $7(1.2 \%)$ \\
Female & $24(4.3 \%)^{*}$ & $4(.7 \%)$ & $11(2.0 \%)$ & $1(.2 \%)$ \\
Sum & $42(4.4 \%)$ & $10(1.0 \%)$ & $23(2.4 \%)$ & $8(.8 \%)$ \\
\hline
\end{tabular}

Note. ${ }^{*} p<.05 ;{ }^{* *} p<.01$ 
The current impacts of different types of child abuse were evaluated by respondents' self-ratings on a scale of 0 to $10(0=$ no effect, $10=$ serious effect $)$. The average current negative effect of emotional child abuse is $4.96(\mathrm{SD}=2.78), 3.94$ $(\mathrm{SD}=2.97)$, and $5.10(\mathrm{SD}=2.70)$ for mild, moderate, and serious abuse, respectively (See Table 4).

Respondents having experienced child physical abuse reported a lower degree of current ill effect than those having experienced child emotional abuse, with average ratings of $2.74(\mathrm{SD}=2.57), 2.86(\mathrm{SD}=2.58)$, and $2.97(\mathrm{SD}=2.73)$ (See Table 5).

In contrast, the current negative influence of child sexual abuse was higher than the other two types proportional to the severity of the abuse, ranging from 4.21 $(\mathrm{SD}=3.04)$ for no-contact abuse to $5.38(\mathrm{SD}=3.53)$ for abuse with penetration (See Table 6).

The emotional, physical, and sexual forms of child abuse were not found to have significantly different current adverse impacts upon the participants in this study. Men reported a higher degree of ill influence for physical abuse than women, especially in the mild and severe categories. This situation was reversed with respect to moderate physical abuse. No gender difference in lasting negative effects was found for emotional or sexual abuse. The results are presented in Tables 4-6.

Table 4. Current effects of child emotional abuse.

\begin{tabular}{cccc}
\hline \multirow{2}{*}{ Gender } & \multicolumn{3}{c}{ Severity of abuse } \\
\cline { 2 - 4 } & Mild & Moderate & Severe \\
\hline Male & $5.26(2.91)$ & $3.69(3.10)$ & $5.36(2.69)$ \\
Female & $4.65(2.62)$ & $4.25(2.80)$ & $4.75(2.72)$ \\
Sum & $4.96(2.78)$ & $3.94(2.97)$ & $5.10(2.70)$ \\
\hline
\end{tabular}

Note. ${ }^{\star} p<.05 ;{ }^{* *} p<.01$.

Table 5. Current effects of child physical abuse.

\begin{tabular}{cccc}
\hline \multirow{2}{*}{ Gender } & \multicolumn{3}{c}{ Severity of abuse } \\
\cline { 2 - 4 } & Mild & Moderate & Severe \\
\hline Male & $3.08(2.81)^{\star *}$ & $2.74(2.50)$ & $3.46(2.67)^{\star *}$ \\
Female & $2.35(2.20)$ & $3.09(2.72)^{\star *}$ & $.67(1.63)$ \\
Sum & $2.74(2.57)$ & $2.86(2.58)$ & $2.97(2.73)$ \\
\hline
\end{tabular}

Note. ${ }^{\star} p<.05 ;{ }^{* *} p<.01$.

Table 6. Current effects of child sexual abuse.

\begin{tabular}{ccccc}
\hline & \multicolumn{4}{c}{ Severity of abuse } \\
\cline { 2 - 5 } Gender & $\begin{array}{c}\text { Mild } \\
\text { (no genital contact) }\end{array}$ & $\begin{array}{c}\text { Mild } \\
\text { (emotional/sexual humiliation) }\end{array}$ & $\begin{array}{c}\text { Moderate } \\
\text { (genital contact) }\end{array}$ & $\begin{array}{c}\text { Severe } \\
\text { (penetration) }\end{array}$ \\
\hline Male & $3.79(3.47)$ & $5.20(3.65)$ & $5.13(3.36)$ & $5.25(3.69)$ \\
Female & $4.43(2.82)$ & $2.75(2.32)$ & $4.25(2.82)$ & $5.60(3.65)$ \\
Sum & $4.21(3.04)$ & $4.11(3.29)$ & $4.69(3.08)$ & $5.38(3.53)$ \\
\hline
\end{tabular}

Note. ${ }^{\star} p<.05 ;{ }^{* *} p<.01$. 
To summarize, the prevalence rates of the mild, moderate, and severe levels of child emotional abuse were $16.4 \%, 8.8 \%$, and $6.9 \%$ respectively, and those of physical abuse were $34.3 \%, 19.3 \%$, and $3.1 \%$, respectively. Regarding child sexual abuse, $4.4 \%$ of the participants reported having had no genital contact and $1.0 \%$ of them reported having experienced emotional humiliation. The prevalence rates of the moderate and severe types were $2.4 \%$ and $.8 \%$. More males were found to suffer from moderate or severe emotional abuse and all levels of physical abuse than females. Compared to males, females suffered more by the moderate physical abuse and mild sexual abuse.

\section{Discussion}

This phenomenological study conducted among Chinese college students 1) estimates the prevalence rates of three types of child abuse (emotional, physical, and sexual abuse) with varied severity (mild, moderate, and severe) in China, and 2) probes the current negative impacts of such abusive experience.

Our results show that child physical abuse occurred most frequently among the three types of abuse in our sample, and sexual abuse was reported least by the participants; emotional abuse rated mid-way between the two. Prevalence rates were inversely related to the severity of abuse for physical and emotional maltreatment; for instance, the prevalence rates of physical abuse: mild $(34.3 \%)>$ moderate $(19.3 \%)>$ severe $(3.1 \%)$. In contrast, sexual abuse was most frequent for the middle category of severity, with mild and severe abuse occurring less often. And we found that different types of child abuse's current influence on one's well-being are different. Child physical abuse had the lowest influence on current sense of well-being while child emotional and sexual types had more serious impact.

\subsection{Prevalence Rates of Child Abuse}

\section{Child Emotional Abuse}

Regarding emotional abuse, $16.4 \%$ of the participants in this study reported that they had been emotionally abused through systematic repression, degradation, or humiliation for a long period of time during their childhood. Of these participants, $8.8 \%$ suffered such abuses as being threatened or forced to restrict their contact with others, or being totally controlled; a smaller number of participants (6.9\%) reported that they had experienced the most severe forms of emotional maltreatment. Overall, no gender difference was observed for mild emotional abuse; however, more males than females experienced moderate and severe levels of emotional abuse.

The estimated prevalence rate of child emotional abuse in our study (22.1\%) was comparable to the rate of $19.6 \%$ reported by Fang et al. in their systematic review on child emotional abuse in China (Fang et al., 2015). Nonetheless, the overall estimate of prevalence in China was not only significantly lower than the prevalence rate of $36.5 \%$ (95\% CI: $26.6 \%$ - 47.6\%) in North America, but also significantly lower than that of $41.6 \%$ (95\% CI: $23.3 \%$ - 62.5\%) in Asia (Stolten- 
borgh et al., 2012).

These disparities may be attributed to at least two possible factors. First, the prevalence of emotional abuse prevalence is indeed low as the result of mainland China's "single-child" policy implemented in the late 1970s and the overall improvement of education. Being the only child at home, one is more likely to enjoy more love, care, and protection, rather than be subject to harsh discipline or punishment from his/her parents, grandparents, and other family members. With the development of Chinese society, more and more parents are better educated and more capable of educating their children in a more enlightened and civilized way. Most of our participants are "the only child" in their family, and hence they may indeed have been less punished or abused by their more educated parents than children of previous generations. The relatively low prevalence in our study thus indicates a real decrease in child emotional abuse in mainland China.

Second, the prevalence of child emotional abuse might have been underestimated in the current study. The higher level of child emotional abuse found in Western countries may be associated with the parenting style or children's interpretation of their parents' behavior in the context of Western culture. Emotional abuse may be perceived as the most extreme manifestation of emotional discipline strategies, which might be more frequently used in collectivist Asian cultures than in individualist Western countries (Stoltenborgh et al., 2011; Hofstede, 2001). However, under the influence of cultural normativeness of these strategies and traditional Chinese culture with filial piety at its core, Chinese people, unlike people from other countries, may be more used to viewing their parents' humiliating or controlling parental acts as conventional ways of discipline rather than forms of abuse (Lansford et al., 2010).

\section{Child Physical Abuse}

Child physical abuse was the most common and frequent type among the three forms of abuse, reaching $38.6 \%$ on average. This number is comparable with the estimate of child physical abuse for China (36.6\%, 95\% CI: 30.4\% 42.7\%) based on a meta-analysis of 47 Chinese studies with participants from mainland China, Hong Kong, and Taiwan (Ji \& Finkelhor, 2015; Tang, 2006). Mild physical abuse was reported by $43.7 \%$ of male participants. In other words, nearly half of the men were once hit, smacked, or held against their will by their parents, elder friends, or even strangers.

However, these figures stand in sharp contrast to the international physical abuse rates and those found in Western countries. For example, the estimated prevalence rate of child physical abuse found in this study (38.6\%) is higher than Stoltenborgh et al.'s (2013a) international estimate (22.6\%, 95\% CI: $19.6 \%$ 26.1\%) as well as their estimates for North America (24.0\%, 95\% CI: $20.1 \%$ 28.5\%), Europe (22.9\%, 95\% CI: $14.9 \%$ - 33.6\%), and Australia (14.3\%, 95\% CI: $7.2 \%-26.5 \%)$.

The overall higher level of socioeconomic development in Western countries may exert a strong positive effect on family environment resulting in lower levels 
of child abuse. Furthermore, the higher prevalence of child physical abuse in China may reflect the conventional punishment-oriented Chinese parenting (Ji \& Finkelhor, 2015). According to the traditional Chinese view, dutiful sons are the product of physical discipline. Some mild or moderate degree of physical abuse is culturally accepted and justified as a necessary and robust way to educate children, especially young boys. Such a tradition or normativeness of physical discipline in Chinese society can bring about a frequent use of this strategy, or a higher prevalence of physical abuse (Lansford et al., 2010).

Regarding severe child physical abuse, males (6.6\%) scored much higher than females $(.9 \%)$ in our sample. Since boys are believed to be physically stronger, more aggressive, and naughtier than girls by nature, they are apt to provoke conflict with others and thus get injured in a tussle, and incur more corporal punishments from family members or teachers. In contrast, girls are often considered as physically weaker and more agreeable in character, thereby needing protection rather than punishment. This may be consistent with our finding that only five girls in our study reported having experienced severe physical abuse.

\section{Child Sexual Abuse}

The prevalence of the mild-level child sexual abuse in our survey was around $5 \%$, while the moderate $(2.4 \%)$ and severe $(.8 \%)$ levels were far less than that. The prevalence rate in our study is lower than the reported rates of other areas, such as Europe and South Africa. For example, the prevalence of child sexual abuse of a community-based sample of 3515 children aged 10 - 17 years $(56.6 \%$ female) in South Africa was 9\%, and the prevalence rates in the Nordic countries were between $3 \%-23 \%$ for boys and $11 \%-36 \%$ for girls before the age of 18 (Kloppen et al., 2016; Meinck et al., 2016).

The low prevalence rates of child sexual abuse in China may be related to the Chinese traditional conservative attitude toward sex and the sense of shame attached to sexuality. Victims of sexual abuse, influenced by their Chinese collectivist consciousness, might not be willing to disclose their abusive experiences for fear of losing their privacy, being stigmatized, or inflicting shame on their family. In addition, Chinese parents' rather high involvement in children's personal affairs including sexuality can be a positive factor protecting children from being sexually abused.

Furthermore, cultural differences in people's conception of sexuality and sexual abuse may also result in differences in the prevalence rates of child sexual abuse. For example, an item in our questionnaire "the child is forced to expose his or her naked body or to watch somebody else expose his or her naked body," can be taken as forms of mild sexual abuse in countries like America, but this is not always the case in the eyes of Chinese people. Therefore, our Chinese participants might have chosen not to report such an event. Similar explanations may be applicable to other types of abuse. Therefore, despite a large difference in rates of child abuse, we cannot exclude the possibility that language, translation, or the original structure of the questionnaire in use limit its effectiveness when applied to participants with different cultural backgrounds. 
In regards to gender, inconsistent with the Hong Kong study (Tang, 2006), the current survey did not find a significant difference between males and females in the prevalence rates of child sexual abuse. Men reported more abuse by an elder acquaintance, whereas women reported more abuse by adult strangers. Interestingly, studies in other countries revealed higher rates of intrafamilial abuse of girls and extrafamilial abuse of boys (Oz \& Ogiers, 2006). If a girl was sexually abused by an acquaintance, the abuser was most likely to be her father or another male caregiver.

\subsection{Current Effects of Child Abuse}

Different types of child abuse can be related to an array of symptoms later in life and this was measured in our study by a self-report of subjective sense of suffering; none of the results were statistically significant. On a range of 0 (do not suffer today) to 10 (suffer terribly today), the results showed a moderate (about 2 5) level of attribution to past abusive experience. Child physical abuse had the lowest influence on current sense of well-being while child emotional and sexual maltreatment had the highest impact.

One interesting finding was that the effect of moderate child emotional abuse on present suffering was lower than that of mild emotional abuse. However, when looking at the items more closely, we see that the situations of moderate emotional abuse, for instance, "someone controlled the child's freedom," seem to be rather common and socially acceptable for Chinese children raised in a nation with a long history of collectivist culture. In case of child sexual abuse, the more severe the abuse was, the more painful the participants reported of their current feeling. For both males and females, severe abuse with genital contact or penetration was the form that did the most harm.

Significant gender difference in respect to the present negative impacts was found only in child physical abuse. Men presently felt much worse than women if they had suffered mild or severe child physical abuse. However, moderate physical abuse led to greater present suffering in women than in men. As discussed above, physical discipline is regarded as an acceptable parenting strategy in the context of traditional Chinese culture. Elders, typically parents and teachers as the legitimate performers of strict education do not intend to leave a residue of pain on the grown male child. Nonetheless, this was the case for moderate levels of abuse only. Frequent physical abuse, even the mild-level abuse inflicted upon Chinese boys could have easily triggered a strong inclination of depression observed in the male participants, even if they seldom question such repeated abuse events in real life.

Moreover, as indicated by this study, girls suffered less severe physical abuse in Chinese culture and society. The moderate level of physical abuse, while rare, caused more negative effects on girls than on boys. Girls may have perceived having been punished unjustly because they believed that, as girls, they should have been treated more gently. Interestingly, severe physical abuse may also be expected to bring a more adverse effect on girls than boys due to the common 
belief that girls should not be given severe corporal punishments like boys. Nonetheless, this belief may lead girls to interpret the physical maltreatment as sanctions for their evil or sin. Therefore, girls would then perceive themselves as deserving the abuse and that the abuse is appropriate punishment. If this is the case, they would be reluctant to blame the abusive experience for negative impacts on their lives and prefer to be "grateful" for having been disciplined harshly.

\section{Conclusion}

To summarize, the high rates of child emotional, physical, and sexual abuse in China are particularly striking since child abuse in any form is likely to have profound adverse effects on children's physical, emotional, and psychological development, with lasting consequences. Furthermore, given the different cultural and social norms between China and Western countries, the prevalence rates of child abuse might not be the same. Less experience of child sexual abuse was reported by Chinese college students than by similar Western participants. However, no difference was found in terms of emotional and physical abuse. Our findings leave interesting room for further cross-cultural discussion and investigation of the interaction between cultural values, historical context, and child-rearing practices in different societies.

The present study has a few limitations. First, the study was conducted with a convenient college student sample which may not be representative, and thus might introduce sources of error into the survey. Several meta-analyses have confirmed that the profile of samples can affect the prevalence of different types of child abuse (Stoltenborgh et al., 2013b; Stoltenborgh et al., 2011). The self-reported prevalence of emotional abuse was significantly higher in college samples $(72.4 \% ; \mathrm{n}=2149)$ than in general population samples $(23.6 \% ; \mathrm{n}=$ 15,392) (Stoltenborgh et al., 2012). Second, the questionnaire was adapted from foreign studies and translated into Chinese, and its items may not align with Chinese reality. This culturally different definition and understanding of child abuse may affect the respondents' feedback. Finally, the study may have underestimated the actual prevalence rates of child abuse in China as it was conducted in the participants' class rather than in a private space. The classroom setup might have lowered the chance for students to respond honestly to shame-inducing questions.

All in all, more comprehensive, accurate, and reliable results of the child abuse in China would be possible if a more Chinese-specific survey among a nationally representative sample of Chinese people, with improved methods of measurements that are more sensitive to culturally relevant issues, could be conducted. Factors like gender, socioeconomic and education backgrounds, as well as cultural interpretation of child abuse, deserve more focus in future research.

\section{Conflicts of Interest}

The authors declare no conflicts of interest regarding the publication of this 
paper.

\section{References}

Amado, B. G., Arce, R., \& Herraiz, A. (2015). Psychological Injury in Victims of Child Sexual Abuse: A Meta-Analytic Review. Psychosocial Intervention, 24, 49-62. https://doi.org/10.1016/j.psi.2015.03.002

Brassard, M. R., \& Donovan, K. L. (2006). Defining Psychological Maltreatment. In M. M. Freerick, J. F. Knutson, P. K. Trickett, \& S. M. Flanzer (Eds.), Child Abuse and Neglect: Definitions, Classifications, and a Framework for Research (pp. 151-197). Paul H. Brookers Publishing.

Calvete, E. (2014). Emotional Abuse as a Predictor of Early Maladaptive Schemas in Adolescents: Contributions to the Development of Depressive and Social Anxiety Symptoms. Child Abuse \& Neglect, 38, 735-746.

https://doi.org/10.1016/j.chiabu.2013.10.014

Chasson, G. S., Mychailyszyn, M. P., Vincent, J. P., \& Harris, G. E. (2013). Evaluation of Trauma Characteristics as Predictors of Attrition from Cognitive-Behavioral Therapy for Child Victims of Violence. Psychological Reports, 113, 734-753.

https://doi.org/10.2466/16.02.PR0.113x30z2

Conway, F., Talreja, P., McCarthy, J., \& Conway, F. (2013). Thought and Language Disorder among Sexually Abused Children in a Psychiatric Hospital. Psychological Reports, 112, 340-352. https://doi.org/10.2174/1573396312666160914193357

Corsello, G., Villani, A., Verrotti, A., Pettoello-Mantovani, M., Fabrizio, G. C., \& Ianniello, F. et al. (2016). Child Abuse and Neglect and Its Psycho-Physical and Social Consequences: A Review of the Literature. Current Pediatric Reviews, 12, 301-310. https://doi.org/10.2174/1573396312666160914193357

Currie, J., \& Widom, C. S. (2010). Long-Term Consequences of Child Abuse and Neglect on adult Economic Well-Being. Child Maltreatment, 15, 111-120. https://doi.org/10.1177/1077559509355316

Dunne, M. P., Chen, J. Q., \& Choo, W. Y. (2008). The Evolving Evidence Base for Child Protection in Chinese Societies. Asia Pacific Journal of Public Health, 20, 267-276. https://doi.org/10.1177/1010539508325047

Fang, X. M., Fry, D. A., Ji, K., Finkelhor, D., Chen, J. Q., Lannen, P., \& Dunne, M. P. (2015). The Burden of Child Maltreatment in China: A Systematic Review. Bulletin of the World Health Organization, 93, 176-185. https://doi.org/10.2471/BLT.14.140970

Hillis, S., Mercy, J., Amobi, A., \& Kress, H. (2016). Global Prevalence of Past-Year Violence against Children: A Systematic Review and Minimum Estimates. Pediatrics, 137, Article No. e20154079. https://doi.org/10.1542/peds.2015-4079

Hofstede, G. (2001). Culture's Consequences: Comparing Values, Behaviors, Institutions, and Organizations across Nations. SAGE Publications.

Hughes, J. R., Busch, K. G., Zagar, R. J., Grove, W. M., \& Arbit, J. (2009). Looking Forward in Records of Youth Abused as Children: Risks for Homicidal, Violent, and Delinquent Offenses. Psychological Reports, 104, 77-101.

https://doi.org/10.2466/PR0.104.1.77-101

Jaffee, S. R., \& Maikovich-Fong, A. K. (2011). Effects of Chronic Maltreatment and Maltreatment Timing on Children's Behavior and Cognitive Abilities. Journal of Child Psychology and Psychiatry, 52, 184-194. https://doi.org/10.1111/j.1469-7610.2010.02304.x

Ji, K., \& Finkelhor, D. (2015). A Meta-Analysis of Child Physical Abuse Prevalence in 
China. Child Abuse \& Neglect, 43, 61-72. https://doi.org/10.1016/j.chiabu.2014.11.011

Kane, F. A., \& Bornstein, R. F. (2018). Unhealthy Dependency in Victims and Perpetrators of Child Maltreatment: A Meta-Analytic Review. Journal of Clinical Psychology, 74, 867-882. https://doi.org/10.1002/jclp.22550

Kim, D. H., Kim, K. I., Park, Y. C., Zhang, L. D., Lu, M. K., \& Li, D. G. (2000). Children's Experience of Violence in China and Korea: A Transcultural Study. Child Abuse \& Neglect, 24, 1163-1173. https://doi.org/10.1016/S0145-2134(00)00175-7

Kloppen, K., Haugland, S., Svedin, C. G., Maehle, M., \& Breivik, K. (2016). Prevalence of Child Sexual Abuse in the Nordic Countries: A Literature Review. Journal of Child Sexual Abuse, 25, 37-55. https://doi.org/10.1080/10538712.2015.1108944

Krug, E. G., Mercy, J. A., Dahlberg, L. L., \& Zwi, A. B. (2002). The World Report on Violence and Health. The Lancet, 360, 1083-1088. https://doi.org/10.1016/S0140-6736(02)11133-0

Lacelle, C., Hébert, M., Lavoie, F., Vitaro, F., \& Tremblay, R. E. (2012). Sexual Health in Women Reporting a History of Child Sexual Abuse. Child Abuse \& Neglect, 36, 247-259. https://doi.org/10.1016/j.chiabu.2011.10.011

Lalor, K., \& McElvaney, R. (2010). Overview of the Nature and Extent of Child Sexual Abuse in Europe. In Council of Europe (Ed.), Protecting Children from Sexual Violence-A Comprehensive Approach (pp. 13-43). Council of Europe.

Lansford, J. E., Malone, P. S., Dodge, K. A., Lei, C., Chaudhary, N., Tapanya, S., \& Deater-Deckard, K. (2010). Children's Perceptions of Maternal Hostility as a Mediator of the Link between Discipline and Children's Adjustment in Four Countries. International Journal of Behavioral Development, 34, 452-461. https://doi.org/10.1177/0165025409354933

Luke, N., \& Banerjee, R. (2013). Differentiated Associations between Childhood Maltreatment Experiences and Social Understanding: A Meta-Analysis and Systematic Review. Developmental Review, 33, 1-28. https://doi.org/10.1016/j.dr.2012.10.001

Madu, S. N. (2001). Prevalence of Child Psychological, Physical, Emotional, and Ritualistic Abuse among High School Students in Mpumalanga Province, South Africa. Psychological Reports, 89, 431-444. https://doi.org/10.2466/pr0.2001.89.2.431

Maniglio, R. (2011). The Role of Child Sexual Abuse in the Etiology of Suicide and Non-Suicidal Self-Injury. Acta Psychiatrica Scandinavica, 124, 30-41. https://doi.org/10.1111/j.1600-0447.2010.01612.x

May-Chahal, C., \& Cawson, P. (2005). Measuring Child Maltreatment in the United Kingdom: A Study of the Prevalence of Child Abuse and Neglect. Child Abuse \& Neglect, 29, 969-984. https://doi.org/10.1016/j.chiabu.2004.05.009

McElvaney, R., \& Lalor, K. (2014). Child Abuse in Europe. In J. Conte (Ed.), Child Abuse and Neglect Worldwide (pp. 27-54). Praeger.

Meinck, F., Cuver, L. D., Boyes, M. E., \& Loening-Voysey, H. (2016). Physical, Emotional and Sexual Adolescent Abuse Victimisation in South Africa: Prevalence, Incidence, Perpetrators and Locations. Journal of Epidemiology and Community Health, 70, 910-916. https://doi.org/10.1136/jech-2015-205860

Norman, R. E., Byambaa, M., De, R., Butchart, A., Scott, J., \& Vos, T. (2012). The long-Term Health Consequences of Child Physical Abuse, Emotional Abuse, and Neglect: A Systematic Review and Meta-Analysis. PLoS Medicine, 9, e1001349. https://doi.org/10.1371/journal.pmed.1001349

Oz, S., \& Ogiers, S. J. (2006). Overcoming Childhood Sexual Trauma: A Guide to Breaking through the Wall of Fear for Practitioners and Survivors: Binghamton. The Ha- 
worth Press.

Pereda, N., Guilera, G., Forns, M., \& Gómez-Benito, J. (2009a). The International Epidemiology of Child Sexual Abuse: A Continuation of Finkelhor (1994). Child Abuse \& Neglect, 33, 331-342. https://doi.org/10.1016/j.chiabu.2008.07.007

Pereda, N., Guilera, G., Forns, M., \& Gómez-Benito, J. (2009b). The Prevalence of Child Sexual Abuse in Community and Student Samples: A Meta-Analysis. Clinical Psychology Review, 29, 328-338. https://doi.org/10.1016/j.cpr.2009.02.007

Pompili, M., Iliceto, P., Innamorati, M., Rihmer, Z., Lester, D., Akiskal, H. S., Girardi, P., Ferracuti, S., \& Tatarelli, R. (2009). Suicide Risk and Personality Traits in Physically and/or Sexually Abused Acute Psychiatric Inpatients: A Preliminary Study. Psycholog ical Reports, 105, 554-568. https://doi.org/10.2466/PR0.105.2.554-568

Runyan, D., Wattam, C., Ikeda, R., Hassan, F., \& Ramiro, L. (2002). Child Abuse and Neglect by Parents and Other Caregivers. In E. G. Krug, J. A. Dahlberg, A. B. Mercy, Z. Lozano, \& R. Lozano (Eds.), World Report on Violence and Health (pp. 57-86). World Health Organization.

Shaffer, A., Huston, L., \& Egeland, B. (2008). Identification of Child Maltreatment Using Prospective and Self-Report Methodologies: A Comparison of Maltreatment Incidence and Relation to Later Psychopathology. Child Abuse \& Neglect, 32, 682-692.

https://doi.org/10.1016/j.chiabu.2007.09.010

Sorbo, M. F., Grimstad, H., Bjorngaard, J. H., Schei, B., \& Lukasse, M. (2013). Prevalence of Sexual, Physical, and Emotional Abuse in the Norwegian Mother and Child Cohort Study. BMC Public Health, 13, Article No. 168.

https://doi.org/10.1186/1471-2458-13-186

Springer, K. W., Sheridan, J., Kuo, D., \& Carnes, M. (2007). Long-Term Physical and Mental Health Consequences of Childhood Physical Abuse: Results from a Large Population-Based Sample of Men and Women. Child Abuse \& Neglect, 31, 517-530. https://doi.org/10.1016/j.chiabu.2007.01.003

Stoltenborgh, M., Bakermans-Kranenburg, M. J., \& Van Ijzendoorn, M. H. (2013a). The Neglect of Child Neglect: A Meta-Analytic Review of the Prevalence of Neglect. Social Psychiatry and Psychiatric Epidemiology, 48, 345-355.

https://doi.org/10.1007/s00127-012-0549-y

Stoltenborgh, M., Bakermans-Kranenburg, M. J., Alink, L. R. A., \& Van Ijzendoorn, M. H. (2012). The Universality of Childhood Emotional Abuse: A Meta-Analysis of Worldwide Prevalence. Journal of Aggression, Maltreatment \& Trauma, 21, 870-890. https://doi.org/10.1080/10926771.2012.708014

Stoltenborgh, M., Bakermans-Kranenburg, M. J., Van Ijzendoorn, M. H., \& Alink, L. R. A. (2013b). Cultural-Geographical Differences in the Occurrence of Child Physical Abuse? A Meta-Analysis of Global Prevalence. International Journal of Psychology, 48, 81-94. https://doi.org/10.1080/00207594.2012.697165

Stoltenborgh, M., Van Ijzendoorn, M. H., Euser, E. M., \& Bakermans-Kranenburg, M. J. (2011). A Global Perspective on Child Sexual Abuse: Meta-Analysis of Prevalence around the World. Child Maltreatment, 16, 79-101. https://doi.org/10.1177/1077559511403920

Swahnberg, K., \& Wijma, B. (2003). The Norvold Abuse Questionnaire (NorAQ): Validation of New Measures of Emotional, Physical, and Sexual Abuse, and Abuse in the Health Care System among Women. European Journal of Public Health, 13, 361-366. https://doi.org/10.1093/eurpub/13.4.361

Tang, C. S. K. (2006). Corporal Punishment and Physical Maltreatment against Children: A Community Study on Chinese Parents in Hong Kong. Child Abuse \& Neglect, 30, 
893-907. https://doi.org/10.1016/j.chiabu.2006.02.012

U.S. Department of Health Human Services (2005). Safe Children and Healthy Families Are a Shared Responsibility: 2005 Community Resource Packet. National Clearinghouse on Child Abuse and Neglect Information.

United Nations Children's Fund (2012). Measuring and Monitoring Child Protection Systems: Proposed Core Indicators for the East Asia and Pacific Region, Strengthening Child Protection Series No. 1. UNICEF East Asia and Pacific Regional Office.

Wijma, B., Schei, B., Swahnberg, K., Hilden, M., Offerdal, K., Pikarinen, U., \& Halmesmäki, E. (2003). Emotional, Physical, and Sexual Abuse in Patients Visiting Gynaecology Clinics: A Nordic Cross-Sectional Study. The Lancet, 361, 2107-2113. https://doi.org/10.1016/S0140-6736(03)13719-1

Wildeman, C., Emanuel, N., Leventhal, J. M., Putnam-Hornstein, E., Waldfogel, J., \& Lee, H. (2014). The Prevalence of Confirmed Maltreatment among US Children, 2004 to 2011. JAMA Pediatrics, 168, 706-713. https://doi.org/10.1001/jamapediatrics.2014.410

Wright, M. O. D., Crawford, E., \& Del Castillo, D. (2009). Childhood Emotional Maltreatment and Later Psychological Distress among College Students: The Mediating Role of Maladaptive Schemas. Child Abuse \& Neglect, 33, 59-68.

https://doi.org/10.1016/j.chiabu.2008.12.007 